\title{
"Electromagnetic" Contribution to the Nucleon Spin
}

\author{
D. Singleton \\ Department of Physics, Virginia Commonwealth University, Richmond, VA 23284-2000
}

(August 20, 2018)

\begin{abstract}
A possible resolution to the question of where the nucleon gets some portion of its internal angular momentum, not carried by the valence quarks, is given in terms of the electromagnetic field angular momentum of the nucleon. This field angular momentum is similiar in origin to that of the Thomson dipole (an electric charge and a magnetic monopole), but arises from the interaction of the quark electric charges and magnetic dipoles. Rough, order of magnitude calculations show that such a field angular momentum could provide some of the nucleon's spin. Under assumptions similiar to those used to calculate hadron mass splittings, QCD should also exhibit a similiar field angular momentum coming from the interaction of color charges with color magnetic dipoles.
\end{abstract}




\section{INTRODUCTION}

In the simple quark model the spin 1/2 nucleon would get its spin from the three valence quarks of which it is constructed. Starting with the European Muon Collaboration experiments [1] it has been realized that the valence quarks are not the only contributors to the total spin of the nucleon. The source of the extra internal angular momentum of the nucleons is taken as coming from virtual quark-antiquark pairs within the nucleon (mainly the strange quarks), or from the gluons. The spin $1 / 2$ of the nucleon can be written out as

$$
\frac{1}{2}=\frac{1}{2} \Delta \Sigma+L_{q}+L_{g}+\Delta G
$$

where $\Delta \Sigma / 2$ is the quark spin contribution, $L_{q}$ and $L_{g}$ are the quark and gluon orbital contributions respectively, and $\Delta G$ is the gluon spin contribution. In this paper we suggest that the contribution from photons should also be included, and we show how a field angular momentum can result from the interaction between the magnetic dipole moments and electric

charges of the valence quarks. Under the assumption that this electrodynamic angular momentum has at least a qualitative counterpart in QCD, we argue that a similiar field angular momentum should arise from the interaction between the color magnetic dipole moments and color charges of the valence quarks.

\section{DIPOLE-CHARGE FIELD ANGULAR MOMENTUM}

To find the field angular momentum contained in the configuration of a magnetic dipole and an electric charge the angular momentum field density should be integrated over all space.

$$
\mathbf{L}_{e m}=\frac{1}{4 \pi} \int \mathbf{r} \times(\mathbf{E} \times \mathbf{B}) d^{3} x
$$

$\mathbf{E}=e \mathbf{r}^{\prime} / r^{\prime 3}$ is the Coulombic electric field of the charged particle, $+e$, located at $\mathbf{R}$ (so $\left.\mathbf{r}^{\prime}=\mathbf{r}-\mathbf{R}\right)$, and $\mathbf{B}=\left(3 \mathbf{r}(\mathbf{r} \cdot \mathbf{M}) / r^{5}\right)-\left(\mathbf{M} / r^{3}\right)$ is the magnetic field of the dipole, $\mathbf{M}$, located at the origin. A quick method to find $\mathbf{L}_{e m}$ directly is to use the result that the 
field angular momentum of a magnetic charge- electric charge system is $\mathbf{L}_{e m}=e g \hat{\mathbf{r}}$, where $+e$ and $+g$ are the electric and magnetic charges respectively, and $\hat{\mathbf{r}}$ is a unit vector which points from the electric charge toward the magnetic charge [2]. Placing a magnetic charge of $+g$ at the origin, a magnetic charge of $-g$ at the point $\mathbf{P}$, and an electric charge of $+e$ at $\mathbf{R}$ yields a field angular momnetum of

$$
\mathbf{L}_{e m}=e\left(-\frac{g \mathbf{R}}{|\mathbf{R}|}+\frac{g(\mathbf{R}-\mathbf{P})}{|\mathbf{R}-\mathbf{P}|}\right)
$$

Expanding $1 /|\mathbf{R}-\mathbf{P}|$ and taking the limit $\mathbf{P} \rightarrow 0, g \rightarrow \infty$, and $g \mathbf{P} \rightarrow-\mathbf{M}$ gives

$$
\mathbf{L}_{e m}=\frac{e \mathbf{M}}{R}-\frac{e \mathbf{R}(\mathbf{M} \cdot \mathbf{R})}{R^{3}}
$$

for the field angular momentum in the dipole-charge system. Directly integrating Eq. (2) for this system again yields the result in Eq. (四). Unlike the field angular momentum of the magnetic charge-electric charge system, which is independent of the distance between the charges, the dipole-charge field angular momentum has a $1 / R$ dependence. Thus as one shrinks the separation between the dipole and the charge this field angular momentum will become increasingly important.

One subtle point with this derivation of the dipole-charge field angular momentum is that the magnetic dipole produced by two monopoles (a Coulombic dipole) is physically different from the magnetic dipole produced by currents (an Amperian dipole). For an Amperian dipole produced by a small current loop in the xy-plane with the current flowing counterclockwise, the magnetic field near the origin will point along the positive z-axis. For a Coulombic dipole to produce the same kind of magnetic field away from the origin, as the previous Amperian dipole, one should place a $+g$ magnetic charge along the positive z-axis and $a-g$ magnetic charge along the negative z-axis. Now, however the magnetic field of the Coulombic dipole will point along the negative z-axis near the origin. Mathematically this is taken into account by adding a delta function term to the standard magnetic dipole field. One adds $+8 \pi \mathbf{M} \delta(\mathbf{r}) / 3$ for the magnetic dipole produced by currents, and $-4 \pi \mathbf{M} \delta(\mathbf{r}) / 3$ for the magnetic dipole produced by monopoles. The limiting procedure for obtaining Eq. 
(四) only produces the $\left(3 \mathbf{r}(\mathbf{r} \cdot \mathbf{M}) / r^{5}\right)-\left(\mathbf{M} / r^{3}\right)$ part of the magnetic dipole field, and not the delta function part. Thus for each of the two cases one should add to Eq. (田) the contribution of this delta function part of the magnetic dipole field. In both cases however the angular momentum density coming from the delta function part of the magnetic field will be of the form

$$
\mathbf{r} \times(\mathbf{E} \times \mathbf{M} \delta(\mathbf{r}))
$$

When this is integrated over all space it will vanish because of the delta function and the factor of $\mathbf{r}$ in the integrand. Thus Eq. (4) is the expression for the field angular momentum for either a magnetic dipole produced by currents or by monopoles.

\section{ELETROMAGNETIC FIELD ANGULAR MOMENTUM OF THE NUCLEON}

We now give a rough estimate of the angular momentum contributed to the nucleon by the electromagentic field, to show that it may be possible that some of the extra internal angular momentum of the nucleon may have an electromagnetic origin. We will consider the proton although similiar considerations apply to the neutron. The state vector for the proton can be written as [3]

$$
\left|p_{\uparrow}\right\rangle=\frac{\epsilon_{i j k}}{\sqrt{18}}\left[u_{i \downarrow}^{\dagger} d_{j \uparrow}^{\dagger}-u_{i \uparrow}^{\dagger} d_{j \downarrow}^{\dagger}\right] u_{k \uparrow}^{\dagger}|0\rangle
$$

where $u^{\dagger}, d^{\dagger}$ are up and down quark creation operators, the arrows represent spin directions, and the latin indices are color degrees of freedom. The part inside the square brackets can be taken as a spin zero "particle" (thus having no magnetic moment) of charge $+e / 3$ which interacts with the remaining spin $1 / 2$ up quark. To get a rough estimate of the electromagnetic field angular momentum contained in a system with a spin zero "particle" of charge $+e / 3$ and a spin $1 / 2$ particle of charge $+2 e / 3$ we take the magnetic dipole of the spin $1 / 2$ particle to be aligned along the positive z-axis and then the magnitude of the $\mathbf{L}_{e m}$ from Eq. (4) is 


$$
\left|\mathbf{L}_{e m}\right|=\sqrt{\mathbf{L}_{e m} \cdot \mathbf{L}_{e m}}=\frac{e M \sin \theta}{3 R}
$$

where $\theta$ is the angle from the z-axis to $\mathbf{R} . \quad M=\left(e_{u} g_{u} / 4 m_{u}\right)$ is the magnitude of the magnetic moment of the up quark, with $g_{u}$ being the electromagnetic gyromagnetic ratio. Taking $\theta=\pi / 2, g_{u} \approx 2, m_{u} \approx 2 \mathrm{MeV} \approx 4 m_{e}, R=0.5$ Fermi $=0.5 \times 10^{-13} \mathrm{~cm}$, and inserting numbers in Eq. (7) (using the units of Ref. [2] and putting a factor of $\hbar / c$ back in $\mathbf{M}$ and a factor of $c$ in the denominator of Eq. (7)) yields $\left|\mathbf{L}_{e m}\right|=0.3(\hbar / 2)$. In this estimate the electromagnetic field angular momentum is on the order of the fundamental unit of angular momentum and therefore could conceivably contribute to the total internal angular momentum of the nucleon. In doing this rough estimate we have taken some of the numbers so as to favour a large electromagnetic angular momentum. Probably the most questionable insert is in the mass of the up quark, where we have taken the lower limit given in Ref. [G], and we have used the current mass rather than the constituent mass. Using the constituent mass would tend to lower the value of the field angular momentum by one or two orders of magnitude thus making it less likely that the electromagnetic field angular momentum plays any significant role in the internal angular momentum of the nucleon. However we have also taken $g_{u} \approx 2$ (which is the order of magnitude value that one gets from indirect fits to observed baryon magnetic moments [3]). If $g_{u}$ were larger than 2 this would help increase the electromagnetic field angular momentum. Also if the separation between the constituents, $R$, inside the nucleon were smaller than our estimate this would tend to increase the electromagnetic field angular momentum. As an aside this is probably why this field angular momentum does not play a significant role in atomic size systems despite the fact that such systems certainly have both charges and magnetic dipole moments : in atomic scale systems the separation, $R$, between the constituents is five orders of magnitude larger which would decrease the field angular momentum by five orders of magnitude. Finally, any sub-quark or sub-lepton models, where the constituents had charges and magnetic moments, would almost certainly have to take this electromagnetic field angular momentum into account, since the experimental upper limit on quark and 
lepton sizes is several orders of magnitude smaller than the fermi size scale of the nucleons.

An objection to this estimate is that we have treated the dipole-charge field angular momentum in a semi-classical way. In some cases this can give accurate results, as in the case of the Thomson dipole where requiring that the field angular momentum equal some integer multiple of $\hbar / 2$ [5] yields the Dirac quantization condition. A more careful analysis of the Thomson dipole [6] shows that the field angular momentum is not a proper quantum mechanical angular momentum when considered by itself, since it does not satisfy the standard commutator $\left[L_{i}, L_{j}\right]=i \epsilon_{i j k} L_{k}$. The same holds for the dipole-charge system : the field angular momentum given in Eq. (2) does not, by itself, satisfy the standard angular momentum commutator. It is straightforward, however, to show that the total angular momentum of the dipole-charge system

$$
\mathbf{J}=(\mathbf{R} \times \mathbf{D})+\mathbf{L}_{e m}
$$

does satisfy $\left[J_{i}, J_{j}\right]=i \epsilon_{i j k} J_{k}$. In Eq. (8), $\mathbf{D}=-i \nabla-e \mathbf{A}$ is the covariant derivative. To show that $\mathbf{J}$ satisfies $\left[J_{i}, J_{j}\right]=i \epsilon_{i j k} J_{k}$ it is necessary to use the fact that the covariant derivative operator does not commute with itself, but rather $\left[D_{i}, D_{j}\right]=i e \epsilon_{i j k} B_{k}$ [6], with $B_{k}$ being the magnetic field.

From the above one might conclude that although it is technically correct to include the electromagnetic field angular momentum in the total internal angular momentum of the nucleon so that Eq. (1) becomes

$$
\frac{1}{2}=\frac{1}{2} \Delta \Sigma+L_{q}+L_{g}+\Delta G+L_{p}+\Delta P
$$

(where the last two terms are the photon orbital and spin contributions respectively) there is only a marginal chance that this is the source of the extra angular momentum. Even with this pessimistic view it is possible that the color version of the above mechanism could be invoked to explain the internal angular momentum of the nucleon. In addition to electric charge, quarks carry a color charge, which when coupled with the spin 1/2 nature of the quarks produces a color magnetic moment. The gauge field part of the QCD angular 
momentum can be written just as in Eq. (2) except with $\mathbf{E} \rightarrow \mathbf{E}^{a}$ and $\mathbf{B} \rightarrow \mathbf{B}^{a}$, where $\mathbf{E}^{a}$ and $\mathbf{B}^{a}$ are the color-electric and color-magnetic fields respectively. As in Ref. [7] one can write the QCD field angular momentum in the following form

$$
\mathbf{L}_{Q C D}=\frac{1}{4 \pi} \int \mathbf{r} \times\left(\mathbf{E}^{a} \times \mathbf{B}^{a}\right) d^{3} x
$$

(The total QCD angular momentum also includes terms for the quark spin and quark orbital angular momentum [7] which we have not written out explicitly here). Except for the color index $a$ this looks identical to the electromagnetic field angular momentum. At this point it is impossible to continue rigorously as in the electromagnetic case, since the strongly coupled, non-linear nature of QCD prevents one from determining the $\mathbf{E}^{a}$ and $\mathbf{B}^{a}$ produced by the quarks at all energy scales. At a large enough energy scale, so that asymptotic freedom would allow one to treat the QCD interaction perturbatively, the non-confining part of the strong interaction potential should look similiar to the electromagnetic interaction potential except for the replacement of $\alpha_{e m} \rightarrow \alpha_{s}$ (i.e. the one-gluon exchange potential would be the QCD version of the Breit-Fermi potential of QED as in Refs. [8] and [3]). Thus under the assumption that one can deal with the one-gluon and confining parts of the color interaction separately, the perturbative part of the color electric and magnetic fields should have the following functional form

$$
\mathbf{E}^{a}=\frac{g T^{a} \mathbf{r}^{\prime}}{r^{3}} \quad \mathbf{B}^{a}=T^{a}\left(\frac{3 \mathbf{r}(\mathbf{r} \cdot \mathbf{M})}{r^{5}}-\frac{\mathbf{M}}{r^{3}}\right)
$$

where $T^{a}$ are the standard $\mathrm{SU}(3)$ matrices, and $\mathbf{M}=g \mathcal{G}_{c} \mathbf{S} / 2 m$ is the color magnetic moment, with $\mathcal{G}_{c}$ being the color equivalent of the electromagnetic g-factor. Inserting these color electric and magnetic fields from Eq. (11) into Eq. (10) we find that the QCD version of the electromagnetic angular momentum of Eq. (4) is

$$
\mathbf{L}_{Q C D}=-\frac{4}{3}\left(\frac{g \mathbf{M}}{R}-\frac{g \mathbf{R}(\mathbf{M} \cdot \mathbf{R})}{R^{3}}\right)
$$

This is similiar to Eq. (4) except for the replacement $e \rightarrow g$ and the factor of $-4 / 3$. Interpreting the proton state vector given in Eq. (6) as a color-anticolor bound state of a spin 
0 and spin $1 / 2$ particles one finds that the sum over the color index $a$ gives $\Sigma_{a=1}^{8} T_{1}^{a} T_{2}^{a}=-4 / 3$ (where 1 and 2 refer to the spin 0 and spin $1 / 2$ particles). The magnitude of this angular momentum is then

$$
\left|\mathbf{L}_{Q C D}\right|=\frac{4 g M \sin \theta}{3 R}
$$

where $M=g \mathcal{G}_{c} / 4 m_{u}$ is the magnitude of the color magnetic moment of the up quark. We take $\theta=\pi / 2$, and $\mathcal{G}_{c} \approx 2$ as in the previous electromagnetic case. Taking $\alpha_{s} \approx 1=137 \alpha_{e m}$ we find that even if we use the constituent mass for $m_{u} \approx 137 \times 2 \mathrm{MeV}=274 \mathrm{MeV}$ (i.e. so the factors of 137 cancel between the coupling and mass terms) that the magnitude of the QCD field angular momentum comes to $\left|\mathbf{L}_{Q C D}\right|=1.2(\hbar / 2)$ relative to the estimate for the electromagnetic case. Within this tree-level approximation for the nonconfining part of the QCD interaction we find that the color field angular momentum will contribute a significant amount to the total spin of the nucleon.

The main objection to the preceding calculation is the lack of a firm theoretical justification for using basically an electromagnetic form for the QCD interaction in a regime where such an approximation is questionable. The use of electromagnetic analogies for low energy QCD is not unprecedented. In Ref. [8] and [3] the mass splittings of QCD bound states (such as the $\Delta^{+}$and proton, or the $\Sigma^{0}$ and $\Lambda$ ) are explained in terms of a color "hyperfine" spinspin interaction. The results are qualitatively and, to an extent, quantitatively accurate. In any case there is certainly some energy scale where the color version of the electromagnetic dipole-charge field angular momentum should be rigourously applicable, and one would expect it to contribute more angular momentum than the electromagnetic effect, due to the presence of group theoretical color factors and also since $\alpha_{s}>\alpha_{e m}$.

As in the electromagnetic case it can be shown that $\mathbf{L}_{Q C D}$ is not a good quantum mechanical angular momentum when considered by itself since it does not satisfy the standard commutation relationship $\left[L_{i(Q C D)}, L_{j(Q C D)}\right]=i \epsilon_{i j k} L_{k(Q C D)}$. It is only the sum of this color field angular momentum with the contribution coming from the quarks that satisfies the proper commutation relationships. This indicates that a more rigorous treatment of either 
the electrodynamic or the chromodynamic systems should take this into account rather than treating the terms separately. This also raises questions about discussing the terms in Eqs. (11) or (9) separately. The difficulties in determining individual terms (e.g. $\left.L_{q}\right)$ in Eqs. (10) or (9), may be connected with the fact that some terms may not be proper quantum mechanical angular momenta when considered separately.

Finally, one possible way to distinguish the two cases (i.e. photons or gluons carrying the extra angular momentum of the nucleon) would be to compare the spin structure of the proton with that of the neutron. The state vector for the neutron is

$$
\left|n_{\uparrow}\right\rangle=\frac{\epsilon_{i j k}}{\sqrt{18}}\left[d_{i \uparrow}^{\dagger} u_{j \downarrow}^{\dagger}-d_{i \downarrow}^{\dagger} u_{j \uparrow}^{\dagger}\right] d_{k \uparrow}^{\dagger}|0\rangle
$$

As with the proton state vector of Eq. (6) one can view this as a bound state of a spin 0 and a spin $1 / 2$ particle. Now, however, the electric charges on the spin 0 and spin $1 / 2$ particles are $+1 / 3$ and $-1 / 3$ respectively, thereby reducing the electromagentic angular momentum of the neutron by a factor of 2 relative to the proton. If the color field angular momentum is the source of the nucleon's extra angular momentum then both the neutron and the proton should carry the same color field angular momentum, since from the state vectors of Eqs. (6) (14) both are similiar color-anticolor bounds states.

\section{DISCUSSION AND CONCLUSIONS}

In this paper we have proposed that the rarely discussed electromagnetic field angular momentum of a magnetic dipole and an electric charge may provide a portion of the nucleon's internal angular momentum not accounted for by the valence quarks. (One source that does briefly mention the dipole-charge system's field angular momentum is Ref. [9]). From a rough, semi-classical estimate we find that this electromagnetic field angular momentum could contribute to the nucleon's spin. This points out that at least from a techincal standpoint Eq. (11) should be expanded to include terms for the photons as in Eq. (9).

Even if the electromagnetic field angular momentum turns out not to be the source of any of nucleon's spin, the color version of this field angular momentum can be postulated 
as the source of the extra internal angular momentum. While this is equivalent to the statement that some of the nucleon's spin comes from the gluons (i.e. the $L_{g}+\Delta G$ terms in Eq. (1)), it gives a more concrete and detailed picture of how this may come about from the interaction of color charges with color magnetic dipoles. This picture also allows one to make definitive predictions about certain states. For example, in this picture the $\pi^{+}=(1 / \sqrt{6})\left[u_{i \uparrow}^{\dagger} \bar{d}_{i \downarrow}^{\dagger}-u_{i \downarrow}^{\dagger} \bar{d}_{i \uparrow}^{\dagger}\right]|0\rangle$ should carry no net field angular momentum in either the electromagnetic or gluonic fields, since the field contributions from the two terms cancel one another. Also, for mesons and bayrons with heavy quarks the field angular momentum should play less of a role, due to the inverse dependence of the magnetic moment on the particle's mass. At large enough energy scales this electromagnetic picture of a field angular momentum arising from the interaction of a dipole and charge should also work for QCD due to asymptotic freedom. At lower energy scales it is not possible to rigorously determine a functional form for the color electric $\left(\mathbf{E}^{a}\right)$ and color magnetic $\left(\mathbf{B}^{a}\right)$ fields produced by the quarks, due to the nonperturbative nature of the QCD interaction. However, there are cases, such as the mass splittings of hadrons with similiar quark content, where the electrodynamic analogy appears to work qualitatively and quantitatively [8] [3] despite the lack of a firm theoretical justification. If the same postulate is applied to the color version of the dipolecharge field angular momentum one finds that this gluonic field angular momentum could contribution significantly to the total spin of the nucleon.

\section{ACKNOWLEDGEMENTS}

I would like to thank Hannalore Roscher and Justin O'Neill for their help and encouragement during the writing of this paper. 


\section{REFERENCES}

[1] J. Ashman et. al., Phys. Lett. B206, 364 (1988); Nucl. Phys. B328, 1 (1989)

[2] J.D. Jackson, Classical Electrodynamics $2^{\text {nd }}$ Edition, (John Wiley \& Sons, 1975) pg. 251

[3] J.F. Donoghue, E. Golowich, and B.R. Holstein, Dynamics of the Standard Model, (Cambridge University Press, 1992), pgs. 284, 322 and 365-370

[4] Review of Particle Properties, Phys. Rev. D56, 1 (1996)

[5] M.N. Saha, Ind. J. Phys. 10, 145 (1936); Phys. Rev. 75, 1968 (1949); H.A. Wilson, Phys. Rev. 75, 309 (1949)

[6] H.J. Lipkin, W.I. Weisberger, and M. Peskin, Ann. Phys. 53, 203 (1969)

[7] X. Ji, Phys. Rev. Lett. 78, 610 (1997);I. Balitsky and X. Ji, Phys. Rev. Lett. 79, 1225 (1997)

[8] A. DeRujula, H. Georgi, and S.L. Glashow, Phys. Rev. D12, 147 (1975)

[9] R.P. Feynman, R.B. Leighton, and M. Sands, The Feynman Lectures on Physics p. 27-8 (Addison-Wesley Publishing Company, 1977) 